\title{
The polio eradication end game: what it means for
} Europe

D Heymann (david.heymann@phe.gov.uk)1,2, Q Ahmed ${ }^{3}$

1. London School of Hygiene and Tropical Medicine, London, United Kingdom

2. Chatham House Centre on Global Health Security, London United Kingdom

3. State University of New York (SUNY) at Stony Brook, New York, United States

Citation style for this article:

Heymann D, Ahmed Q. The polio eradication end game: what it means for Europe. Euro Surveill. 2014;19(7):pii=20702. Available online: http://www. eurosurveillance.org/ViewArticle.aspx?Articleld=20702

Article submitted on 5 February 2014 / published on 20 February 2014

This edition of Eurosurveillance provides a series of articles related to polio that present a microcosm of some of the issues that have plagued polio eradication since the programme first began, and it also provides many of the solutions.

Had these and other issues been clearly understood in 1988 when the World Health Assembly passed the resolution that committed all countries to polio eradication by the year 2000 , the decision to eradicate would have been more difficult. But fortunately, buoyed by the then recent success in the eradication of smallpox, active debate on whether to use inactivated or live polio vaccines, awareness that many countries had already interrupted polio transmission, and unawareness of the difficult eradication end game, the resolution was passed by consensus of the World Health Organization (WHO)'s member states [1]. Although progress towards eradication has been slower than anticipated in 1988 , paralytic polio has decreased from an estimated 1,000 children per day during 1988 , to approximately 400 during 2013. Today there remain only three countries with endemic polio, Afghanistan, Nigeria and Pakistan, and the recent risk assessment from the European Centre for Disease Prevention and Control (ECDC) reminds us that Europe must remain vigilant with strong surveillance and sustained laboratory capacity [2].

The series of polio articles in this edition begins with the article by Hindiyeh et al. [3] describing direct sewage testing for wild poliovirus antigen, using a multiplex quantitative reverse-transcription PCR (qRT-PCR) for rapid detection of the virus, directly on concentrated sewage samples. When compared to cell culture of the same sewage specimens, which is the initial process in the gold standard testing protocol for confirmation of polio, sensitivity and specificity of the multiplex system were shown to be high. Results were obtained in 24 to 48 hours, rather than the usual five to seven days required for the culture-based protocol.

The time from collection of a stool sample to analysis for polio virus in polio eradication programmes has often been weeks, not days, causing delays in response, more widespread transmission, and greater and more costly containment efforts. Recently though, times from specimen collection to outbreak control have decreased considerably by strengthening transport systems from the field to the laboratory, and modifying the testing protocol [4]. At the same time, there is active research and development of new testing algorithms that can provide more rapid results [5]. Hindiyeh et al. have concluded that their qRT-PCR system could be a promising application for testing of RNA extracted directly from processed stool samples from children with acute flaccid paralysis (AFP), and it remains to be seen whether further study will be conducted along these lines [3].

The article by Manor et al. [6] describes the discovery of a silent introduction of wild poliovirus, in the absence of detection of AFP in children (the standard surveillance methodology), by what they describe as an early warning system of sewage monitoring for poliovirus. They point out that this silent introduction occurred in a highly immune population in which inactivated polio vaccine (IPV) has been used exclusively since 2005, and that AFP surveillance alone had not detected this introduction and circulation. The authors suggest that there is a fundamental role for environmental surveillance in routine monitoring as an early warning system in polio-free countries, possibly more sensitive than surveillance for AFP. Shulman et al. [7] add greater perspective in their report on genetic sequencing of these wild polioviruses. It suggests that they were linked to strains that were circulating in South Asia and Egypt in 2012 and concludes that there had been one, or perhaps more than one, importation event.

Indeed, environmental monitoring in sewage has been used by many countries during the past decade, and it has identified wild poliovirus imported in 2007 to Switzerland from Chad, and in 2013 to Egypt from Pakistan [8,9]. Environmental surveillance has been a mainstay of polio eradication in several developing countries as well, for example in Egypt and India, and 
its wider role in the polio eradication end game continues to be assessed [10].

Van der Maas et al. [11] and Yakovenko et al. [12] discuss the importance of maintaining high polio immunity levels in order to prevent re-establishment of circulation of wild poliovirus, and the vulnerability of countries with lower than optimal population immunity in the general population or with pockets of low coverage such as those in certain religious and other groups. They clearly call attention to the fact that the circulation of wild poliovirus in one country is a threat to all others, and that vaccination coverage, using either IVP or oral polio vaccine (OPV), must be maintained until the circulation of all wild poliovirus has been interrupted. Yakovenko et al. also underscore the fact that adults are at risk from imported polio virus, having isolated wild poliovirus from adults with AFP in the Tajikistan outbreak [12].

A recent polio outbreak also provided a clear demonstration that adults are at risk of paralytic polio during outbreaks. Because adult populations in Namibia had not been vaccinated against polio in the period before independence, and had not developed immunity by exposure to poliovirus because of high quality water and sanitation systems, an importation of wild poliovirus led to an outbreak of paralytic polio in adults in 2006 [13].

Other issues that have been important to polio eradication such as circulating vaccine derived poliovirus (cVDPD) are not discussed in this series, although the ease with which the poliovirus recombines in nature was demonstrated by studies of viral sequences in the Tajikistan outbreak [12]. However, this omission from the series does not minimise the importance of cVDPV as a challenge to polio eradication that the end game will take into account [14]. The decision in Israel to reintroduce OPV after failure to interrupt transmission with IPV, foretells the complexity the end game will face in the event of a reintroduction after eradication has occurred. Outbreak containment strategies for all countries are actively being assessed for application during the post-eradication period [15].

Although solutions to most of the technical problems in polio eradication are either available or under development, risk communication and gaining trust in polio vaccination in the absence of paralytic disease remain a major challenge. This is clearly demonstrated by the experience described by Kaliner et al. [16] in developing trust and paving the way for the supplementary immunisation activities that reintroduce OPV several years after having switched to IPV in routine vaccination programmes.

The importance of trust and risk communication has likewise been clearly demonstrated in the past, when in 2003 polio vaccination was stopped in northern Nigeria because of false rumours, many of which were circulating on the world wide web, that the vaccine was being used in a plot by some Western nations to permanently sterilise young Muslim girls, and in some instances that it was associated with the introduction of AIDS [17]. Although there may have been other reasons than concern over vaccine safety, the governor of one northern state in Nigeria interrupted polio eradication activities, and other northern Nigerian states followed. Within months, polio had spread from Nigeria to neighbouring countries, to Saudi Arabia and Yemen, and from there on to Indonesia [18].

All of the countries affected were members of the Organization of the Islamic Conference who, at their October 2003 summit in Malaysia, adopted a resolution that endorsed and promoted stronger polio eradication activities [19]. Religious leaders became involved as well, and promoted polio vaccination through a series of fatwas and other declarations. Countries that had been free of polio, again had children paralysed by poliovirus, and increased their surveillance and conducted supplementary immunisation activities to prevent the virus from becoming endemic again. They were successful in containing wild poliovirus and interrupting its transmission, but at great financial cost to the countries and the polio partnership.

This series of articles on polio is a timely reminder that polio eradication has not yet been completed, and they confirm that eradication is technically feasible. But obstacles to polio eradication remain. Killings of polio workers in Pakistan and northern Nigeria have caused fear among polio workers, and pose a risk to the life of those who vaccinate door to door. These incidents have prompted a call for action from the Muslim world to counter opposition to the polio eradication programme recently published in The Lancet [20].

Civil unrest, such as that caused by the killing of polio workers, has occurred in the past, but was never targeted specifically at polio eradication. In Sudan in 2005, for example, the United Nations called for days of tranquillity so that polio and other vaccinations could continue [21]. But the solution to violence targeted at the global eradication of polio and at vaccination programmes in general, requires more than vaccine supplies, door-to-door vaccination, and meticulous surveillance. It requires collective ownership and solidarity by all countries, and it may need a prominent and accepted figure in all countries where opposition has been observed, to step forward as a leader and bring polio eradication to completion.

In the meantime, European countries must continue to maintain high levels of polio vaccination coverage, and sustained surveillance of AFP with laboratory support, in order to ensure that wild poliovirus, if imported, is rapidly detected and completely contained. 


\section{Conflict of interest}

None declared

\section{References}

1. Forty-first World Health Assembly. Global eradication of poliomyelitis by the year 2000. Resolution WHA 41.28. Geneva: World Health Organization; 1988. Available from: http:// www.polioeradication.org/content/publications/19880513_ resolution.pdf

2. European Centre for Disease Prevention and Control (ECDC). Risk of introduction and transmission of wild-type poliovirus in EU/EEA countries following events in Israel and Syria - updated risk assessment. Stockholm: ECDC; 2013. Available from: http://www.ecdc.europa.eu/en/publications/ Publications/poliomyelitis-risk-assessment-update-10December-2013.pdf

3. Hindiyeh MY, Moran-Gilad J, Manor Y, Ram D, Shulman LM, Sofer D, Mendelson E. Development and validation of a rea time quantitative reverse transcription-polymerase chain reaction (qRT-PCR) assay for investigation of wild poliovirus type 1-South Asian (SOAS)strain reintroduced into Israel, 2013 to 2014 . Euro Surveill. 2014;19(7): pii=20710.

4. Global detection of wild and vaccine-derived polioviruses, January 2008-June 2009. Wkly Epidemiol Rec. 2009;84(36):366-70.

5. The Global Polio Eradication Initiative. New lab procedures more reliably detect VDPVs and reduce time to confirm poliovirus by $50 \%$. The Polio Pipeline. 2011; 8. Geneva: World Health Organization; 2011. Available from: http://www. polioeradication.org/Research/PolioPipeline/No8Summer2011. aspx

6. Manor Y, Shulman LM, Kaliner E, Hindiyeh M, Ram D, Sofer D, Moran Gilad J, Lev B, Grotto I, Gamzu R, Mendelson E. Intensified environmental surveillance supporting the response to wild poliovirus type 1 silent circulation in Israel, 2013 . Euro Surveill. 2014;19(7): $\mathrm{pii}=20708$.

7. Shulman LM, Gavrilin E, Jorba J, Martin J, Burns CC, Manor Y, Moran Gilad J, Sofer D, Hindiyeh MY, Gamzo R, Mendelson E, Grotto I, for the Genotype - Phenotype Identification (GPI) group. Molecular epidemiology of silent introduction and sustained transmission of wild poliovirus type 1, Israel, 2013. Euro Surveill. 2014;19(7):pii=20709.

8. World Health Organization Regional Office for Europe (WHO/ Europe). Wild poliovirus isolated in Switzerland's sewer system; insignificant risk of outbreak. Euro Polio Page. Monthly AFP surveillance bulletin, November 2007 Copenhagen WHO/Europe; 2007. Available from: http://www.euro.who. int/_data/assets/pdf_file/0005/79394/E200711_PolioPage. pdf

9. World Health Organization Office for the Eastern Mediterranean (WHO/Eastern Mediterranean). Importation of wild poliovirus detected in environmental (sewage) samples in Egypt . Cairo: WHO/Eastern Mediterranean; 2011. Available from: http://www.emro.who.int/polio/polio-news/new-poliovirus-egypt.html

10. World Health Organization (WHO). Guidelines for environmental surveillance of poliovirus circulation. Geneva: WHO; 2013. Available from: http://whqlibdoc.who.int/ hq/2003/who_v\&b_03.03.pdf

11. van der Maas NA, Mollema L, Berbers GA, van Rooijen DM, van der Avoort HG, Conyn-Van Spaendonck MA, de Melker HE, van der Klis FR. Immunity against poliomyelitis in the Netherlands, assessed in 2006 to 2007: the importance of completing a vaccination series. Euro Surveill. 2014;19(7): pii=20705.

12. Yakovenko ML, Gmyl AP, Ivanova OE, Eremeeva TP, Ivanov AP, Prostova MA, Baykova OY, Isaeva OV, Lipskaya GY, Shakaryan AK, Kew OM, Deshpande JM, Agol VI. The 2010 outbreak of poliomyelitis in Tajikistan: epidemiology and lessons learnt. Euro Surveill. 2014;19(7):pii=20706.

13. Centers for Disease Control and Prevention (CDC): Outbreak of polio in adults - Namibia, 2006. MMWR Morb Mortal Wkly Rep. 2006;55(44):1198-201.

14. The Global Polio Eradication Initiative. Post-eradication preparing for a lasting polio-free world. The Polio Pipeline. 2011; 8. Geneva: World Health Organization; 2011. http://www. polioeradication.org/Research/PolioPipeline/No8Summer2011. aspx

15. The Global Polio Eradication Initiative. Research. Geneva: World Health Organization. [Accessed: 17 Feb 2014]. Available from: http://www.polioeradication.org/Research.aspx

16. Kaliner E, Moran-Gilad J, Grotto I, Somekh E, Kopel E, Gdalevich M, Shimron E, Amikam Y, Leventhal A, Lev B, Gamzu
R. Silent reintroduction of wild-type poliovirus to Israel, 2013 - risk communication challenges in an argumentative atmosphere . Euro Surveill. 2014;19(7): $\mathrm{pii}=20703$.

17. Kaufman JR, Feldbaum H. Diplomacy and the polio immunization boycott in Northern Nigeria. Health Af (Millwood). 2009;28(4):1091-101. http://dx.doi.org/10.1377/ hlthaff.28.4.1091

18. Polio case count 2003. Geneva: World Health Organization. [Accessed: 17 Feb 2014]. Available from: http://apps.who. int/immunization_monitoring/en/diseases/poliomyelitis/ afpextract.cfm

19. Tenth Session of the Islamic Summit Conference. Resolution no. $14 / 10-S \& T(I S)$. On global cooperation in polio eradication programme among OIC Member States. Putrajaya: Islamic Summit Conference; 2003. Available from: http://www. polioeradication.org/content/publications/20031017_ resolution.pdf

20. Ahmed QA, Nishtar S, Memesh ZA. Poliomyelitis in Pakistan: time for the Muslim world to step in. Lancet. 2013;381(9877):1521-3. http://dx.doi.org/10.1016/ S0140-6736(13)60764-3

21. UN Advance Mission in Sudan. The UN call for days of tranquility bears fruit; more than five million children have been vaccinated against poliomyelitis in Sudan. ReliefWeb. Press release 16 Jan 2005. Available from: http://reliefweb.int/ report/sudan/un-call-days-tranquility-bears-fruit-more-fivemillion-children-have-been-vaccinated 